\title{
The physiological effect of pre-soaking with tryptophan on sugar beet (Beta vulgaris L.) productivity under different levels of salinity stresses
}

\author{
M. Hozayn ${ }^{1}$, A. A. Abd-Elmonem²,3 and Ghada M. Samaha ${ }^{1 *}$
}

\begin{abstract}
Background: Salinity has a negative effect on the productivity of many crops. Therefore, the present study was conducted to investigate the effect of pre-soaking in different concentrations of tryptophan (0.0, 2.5, and 5.0 mM) on growth, yield and root quality of two sugar beet cultivars (DS-9004 and LP-15) under different salinity levels (320, 2000, 4000, 6000, and 8000 ppm) in an effort to reduce salinity damage on sugar beet. The data were collected at 2 stages of growth (75 and 180 days after sowing).

Results: The results revealed that the pre-soaking in tryptophan treatments $(2.5 \mathrm{mM})$ were the most effective treatments under different salinity levels and caused significant increases in all tested parameters of this study. Moreover, increasing salinity levels decreased significantly the root purity parameters and increased impurity parameters of sugar beet. The DS9004 cultivar gave the highest values of all studied parameters compared with the LP-15 cultivar under different salinity levels with tryptophan $(2.5 \mathrm{mM})$. Chlorophyll a content and chlorophyll a/b ratio, as well as carotenoids content, were highest in DS-9004 cultivar, while chlorophyll b content was highest in LP-15 cultivar at 75 days.

Conclusions: Salinity level up to 320 ppm positively affected the growth yield of sugar beet while the highest significant reduction was associated with increasing salinity up to 8000 ppm. Thus, pre-soaking in tryptophan (2.5 mM) has a promotive effect on increasing sugar beet yield under water salinity conditions.
\end{abstract}

Keywords: Salinity, Sugar beet, Pre-soaking, Tryptophan, Growth, Yield, Root quality

\section{Background}

Sugar beet (Beta vulgaris L.) family Chenopodiaceae is one of the world's leading sugar crops. It is the second main source of sugar after sugar cane, it contains sucrose up to $21 \%$ (Memon et al., 2004). Worldwide, the area of sugar beet cultivated, total production (ton) and average root yield (ton ha ${ }^{-1}$ ) reached to 4.47 million ha, 266.8 million tons, and 59.6 ton ha ${ }^{-1}$ (FAOSTAT, 2016). Sugar beet has stronger salt-tolerant characteristics than other crops.

\footnotetext{
*Correspondence: ghka_sam@yahoo.com

${ }^{1}$ Field Crop Research Department, Agricultural and Biological Research Division, National Research Centre, 33 El-Behouth St., (Former El-Tahrir St.) Dokki, Giza 12622, Egypt

Full list of author information is available at the end of the article
}

It is a dicotyledonous plant with high economic value in many countries. In Egypt, the sugar industry depends mainly on sugar cane and sugar beet crops. The total production of sugar beet in 2016 was about 13,323,369 tons with an area of 254,991 ha with an average root yield of $52.3 \mathrm{ton} / \mathrm{ha}^{-1}$ (ha $=2.38 \mathrm{fed}$ ). Sugar beet produced 1.25 million tons of sugar represented about $50 \%$ of the local production (FAOSTAT, 2016).

Salinity stress is a major limitation of global crop production. Soil salinity is adversely affecting physiological and metabolic processes, finally diminishing growth and yield (Hozayn et al., 2019). Excessive salts injure plants by disturbing the uptake of water into roots and interfering with the uptake of competitive nutrients (Franzen, 
2007). Reduction in plant growth has also been attributed to reduce water absorption and osmotic effect, nutritional deficiency on account of ionic imbalance and decrease in many metabolic activities (Rezaee et al., 2012). Several studies have reported that salinity hurts hormones hence germination (Debez et al., 2001).

Amino acids play an important role in plant tolerance to salinity conditions, amino acids act as antioxidants or activators of phytohormones which increases the productivity and quality of sugar beet (El-Gamal et al., 2016). Many previous studies which reported that tryptophan is one of the amino acids that play a vital role for improving plant growth (El-Bassiouny, 2005) through the availability of nutrients and water (Abbas et al., 2013) protects plants (Hussein et al., 2014) regulating ion transport and remove toxic substances of heavy metals (Rai, 2002). Spraying plant leaves with tryptophan stimulate growth regulators and photosynthesis that reflected improves plant growth (Bakry et al., 2016). As the performance of pre-soaked seeds in tryptophan is much better than untreated under salinity conditions (Saranya, 2017). Therefore, this study aimed to use the pre-soaking with tryptophan to alleviate the negative effects of salinity stress conditions and improve sugar beet yield and quality.

\section{Materials and methods}

A pot experiment was performed at the screen greenhouse of Field Crops Research Department, National Research Centre, Dokki, Giza, Egypt, to study the effect of pre-soaking of two cultivars (DS-9004 and LP-15) sugar beet seeds (Table 1 ) in different concentrations of tryptophan $(0.0,2.5$, and $5.0 \mathrm{mM})$ on growth, yield, and root quality of sugar beet plants grown under different salinity levels (320, 2000, 4000, 6000, and 8000 ppm). Sugar beet seeds were soaked in different tryptophan concentrations for $6 \mathrm{~h}$ before sowing. The treatments were arranged in a split-plot in CRD design where salinity levels and tryptophan concentrations were allocated in the main and sub-plot, respectively. The sugar beet soaked seeds were sown in pots $(50 \mathrm{~cm}$ in diameter) containing equal amounts of sand and clay (1:1) soil. Each treatment consisted of 4 replicates. The pots were irrigated with equal volumes of the various salinity levels after 21 days from sowing. Irrigation was run as follows 3 times

Table 1 Origin and sources of sugar beet (Beta vulgaris L.) cultivars used in this study

\begin{tabular}{lll}
\hline & Cultivars & \\
\cline { 2 - 3 } & DS-9004 & LP-15 \\
\hline Seeds & Multigerm & Multigerm \\
Origin & Denmark & France \\
Source & aSCRI & $*$ SCRI \\
\hline
\end{tabular}

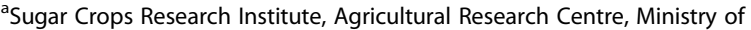
Agriculture, Giza, Egypt with saline solutions and one with tap water. NPK fertilization was done as the recommended rate. After 15 days from sowing, thinning was carried out, so as two uniform seedlings were left in each pot for studying the effect of different treatments on the yield and quality of sugar beet cultivars under different treatments.

After 75 days from sowing, one plant was taken from each pot to determine root length and diameter $(\mathrm{cm})$, fresh and dry weight (g). Photosynthetic pigments in leaves were determined using a spectrophotometer. At harvest, a sample of fresh roots was taken from each treatment and sent to the Beet Laboratory at Nubaria Sugar Factory to determine root quality. Alpha-amino nitrogen $(\alpha$-amino- $\mathrm{N})$, sodium $(\mathrm{Na})$, and potassium $(\mathrm{K})$ concentrations were estimated according to the procedure of Sugar Company by Auto Analyzer described by Cooke and Scott (1993). TSS and sucrose percentage estimated in fresh samples of sugar beetroot by using a saccharometer according to the method described by AOAC (1995). Sugar loss was calculated using the following formula: Sugar loss $\%=0.29+0.343(\mathrm{~K}+\mathrm{Na})+0.094 \alpha$-amino-N, Sugar recovery \% was calculated using the following equation: Sugar recovery $(\%)=$ sucrose $(\%)$ - sugar loss (\%) (Cooke and Scott, 1993). Recoverable sugar yield $\left(\mathrm{g} \mathrm{plant}^{-1}\right.$ ) was calculated using the following equation of Mohamed (2002): Recoverable sugar yield $=$ root yield $\left(\mathrm{g} \mathrm{plant}^{-1}\right) \times$ sugar recovery. Quality index was calculated as (sugar recovery $(\%) \times 100) /$ sucrose (\%). Gross sugar yield (g plant $\left.^{-1}\right)=$ root yield $\left(\right.$ g plant $\left.^{-1}\right) \times$ sucrose $^{(\%)}$. Sugar loss yield was computed as root yield $\left(\mathrm{g} \mathrm{plant}^{-1}\right) \times$ sugar loss.

The values of all parameters were statistically analyzed to find out the level of significance using MSTAT-C package program (MSTAT-C, 1983). The mean differences were compared by the least significant difference test (LSD) at $5 \%$ level of significance.

\section{Results}

Effect of pre-soaking with tryptophan on root parameters of sugar beet at 75 days after sowing under different salinity levels

Data in Table 2 clearly indicated that pre-soaking sugar beet seeds with tryptophan $(2.5 \mathrm{mM})$ under different salinity levels increased significantly sugar beetroot parameters, i.e., root length and diameter $(\mathrm{cm})$, root fresh and dry weight (g) at 75 days after sowing as compared with tryptophan 0.0 and $5.0 \mathrm{mM}$. On the other hand, with an increase in the salinity of irrigation water up to 8000 $\mathrm{ppm}$, there was a decrease in the values of all these parameters. In all the cases, the increment in root parameters in DS-9004 cultivar was more than LP-15 cultivar as roots length $(18.77 \mathrm{~cm})$, root diameter $(6.10 \mathrm{~cm})$, and root fresh weight $(9.83 \mathrm{~g})$ with tryptophan $(2.5 \mathrm{mM})$ under salinity level at $320 \mathrm{ppm}$. Whilst, LP-15 cultivar was suppressed in root dry weight $(2.99 \mathrm{~g})$ with tryptophan 
Table 2 Effect of pre-soaking two sugar beet seed cultivars with tryptophan and irrigated with different levels of salinity on root parameters at 75 days after sowing

\begin{tabular}{|c|c|c|c|c|c|c|c|c|c|}
\hline \multicolumn{2}{|l|}{ Treatments } & \multicolumn{8}{|c|}{ Root parameters } \\
\hline \multirow[t]{2}{*}{ Salinity (ppm) } & \multirow{2}{*}{$\begin{array}{l}\text { Try. } \\
\text { (mM) }\end{array}$} & \multicolumn{2}{|c|}{ Length $(\mathrm{cm})$} & \multicolumn{2}{|c|}{ Diameter $(\mathrm{cm})$} & \multicolumn{2}{|c|}{ Fresh wt. (g) } & \multicolumn{2}{|l|}{ Dry wt. (g) } \\
\hline & & DS-9004 & LP-15 & DS-9004 & LP-15 & DS-9004 & LP-15 & DS-9004 & LP-15 \\
\hline \multirow[t]{3}{*}{320} & 0.0 & 18.00 & 15.50 & 5.57 & 4.67 & 6.53 & 4.60 & 1.70 & 1.58 \\
\hline & 2.5 & 18.77 & 16.67 & 6.10 & 5.43 & 9.83 & 7.23 & 2.61 & 2.99 \\
\hline & 5.0 & 18.33 & 16.17 & 5.97 & 5.13 & 8.87 & 6.17 & 2.39 & 2.50 \\
\hline \multirow[t]{3}{*}{2000} & 0.0 & 16.83 & 13.53 & 5.20 & 4.20 & 6.43 & 4.50 & 1.67 & 1.57 \\
\hline & 2.5 & 17.71 & 16.63 & 6.00 & 4.87 & 9.17 & 6.37 & 2.58 & 2.60 \\
\hline & 5.0 & 16.97 & 15.50 & 5.73 & 4.67 & 7.90 & 5.00 & 1.86 & 1.84 \\
\hline \multirow[t]{3}{*}{4000} & 0.0 & 16.10 & 13.30 & 5.17 & 3.73 & 5.23 & 3.60 & 1.41 & 1.38 \\
\hline & 2.5 & 17.33 & 15.67 & 5.89 & 4.40 & 7.63 & 5.80 & 2.34 & 1.99 \\
\hline & 5.0 & 16.07 & 14.17 & 5.73 & 4.00 & 6.62 & 4.83 & 2.09 & 1.77 \\
\hline \multirow[t]{3}{*}{6000} & 0.0 & 14.00 & 13.07 & 4.87 & 3.67 & 4.13 & 2.20 & 1.08 & 0.69 \\
\hline & 2.5 & 16.50 & 14.50 & 5.77 & 4.60 & 7.03 & 4.17 & 2.58 & 1.62 \\
\hline & 5.0 & 15.07 & 13.83 & 5.50 & 3.90 & 6.00 & 4.10 & 1.38 & 1.61 \\
\hline \multirow[t]{3}{*}{8000} & 0.0 & 11.50 & 10.50 & 3.40 & 2.93 & 2.13 & 1.13 & 0.66 & 0.37 \\
\hline & 2.5 & 13.17 & 13.33 & 3.99 & 3.23 & 3.20 & 1.80 & 1.92 & 0.69 \\
\hline & 5.0 & 12.67 & 12.83 & 3.67 & 3.17 & 2.83 & 1.23 & 0.99 & 0.47 \\
\hline \multicolumn{2}{|l|}{$\mathrm{LSD}_{5 \%}$} & 2.81 & & 1.26 & & 2.48 & & 0.01 & \\
\hline \multicolumn{10}{|c|}{ Mean of main effects } \\
\hline \multirow[t]{2}{*}{ Variety } & DS-9004 & 15.93 & & 5.24 & & 6.24 & & 1.82 & \\
\hline & LP-15 & 14.35 & & 4.17 & & 4.18 & & 1.58 & \\
\hline \multicolumn{2}{|l|}{$\operatorname{LSD}_{5 \%}$} & ns & & a & & a & & a & \\
\hline \multirow[t]{5}{*}{ Salinity (ppm) } & 320.0 & 17.24 & & 5.48 & & 7.21 & & 2.30 & \\
\hline & 2000.0 & 16.20 & & 5.11 & & 6.56 & & 2.02 & \\
\hline & 4000.0 & 15.44 & & 4.82 & & 5.62 & & 1.83 & \\
\hline & 6000.0 & 14.50 & & 4.72 & & 4.61 & & 1.49 & \\
\hline & 8000.0 & 12.33 & & 3.40 & & 2.05 & & 0.85 & \\
\hline \multicolumn{2}{|l|}{$\mathrm{LSD}_{5 \%}$} & ns & & 0.92 & & 1.81 & & 0.01 & \\
\hline \multirow[t]{3}{*}{ Try. (mM) } & 0.0 & 11.86 & & 3.62 & & 3.37 & & 1.01 & \\
\hline & 2.5 & 13.36 & & 4.19 & & 5.19 & & 1.83 & \\
\hline & 5.0 & 12.63 & & 3.96 & & 4.46 & & 1.41 & \\
\hline \multicolumn{2}{|l|}{ LSD $_{5 \%}$} & ns & & ns & & 1.36 & & 0.04 & \\
\hline
\end{tabular}

ns non significant

${ }^{\mathrm{a}}$ Significant

$(2.5 \mathrm{mM})$ under salinity level at $320 \mathrm{ppm}$. Maximal root parameters were recorded with tryptophan $(2.5 \mathrm{mM})$ under salinity level at $320 \mathrm{ppm}$ for DS-9004 cultivar.

Effect of pre-soaking with tryptophan on photosynthetic pigment contents under different salinity levels at $\mathbf{7 5}$ days after sowing Photosynthetic pigment

The results in Table 3 indicated the pre-soaking in tryptophan $(2.5 \mathrm{mM})$ had increased significantly each of chlorophyll a, b, and carotenoids contents compared with other treatments (tryptophan 0.0 and 5.0 $\mathrm{mM}$ ) at 75 days after sowing under salinity level at $320 \mathrm{ppm}$. Therefore, the highest value of chlorophyll a $(15.62 \mathrm{mg} / \mathrm{g}$ f.w.) was obtained with tryptophan $(2.5 \mathrm{mM})$ for DS-9004 cultivar. While the highest value of chlorophyll b $(7.04 \mathrm{mg} / \mathrm{g}$ f.w.) with tryptophan $(2.5 \mathrm{mM})$ for LP-15 cultivar. On the other hand, chlorophyll a/b ratio insignificantly affected by the pre-soaking treatments under different salinity 
Table 3 Effect of pre-soaking two sugar beet seed cultivars with tryptophan and irrigated with different salinity levels on photosynthetic pigment contents ( $\mathrm{mg} / 100 \mathrm{~g}$ fresh weight) at 75 days after sowing

\begin{tabular}{|c|c|c|c|c|c|c|c|c|c|}
\hline \multicolumn{2}{|l|}{ Treatments } & \multicolumn{8}{|c|}{ Photosynthetic pigment (mg/100 g fresh weight) } \\
\hline \multirow[t]{2}{*}{ Salinity (ppm) } & \multirow{2}{*}{$\begin{array}{l}\text { Try. } \\
\text { (mM) }\end{array}$} & \multicolumn{2}{|l|}{ Chl. (A) } & \multicolumn{2}{|l|}{ Chl. (B) } & \multicolumn{2}{|c|}{ Chl. (A)/(B) } & \multicolumn{2}{|c|}{ Carotenoids } \\
\hline & & DS-9004 & LP-15 & DS-9004 & LP-15 & DS-9004 & LP-15 & DS-9004 & LP-15 \\
\hline \multirow[t]{3}{*}{320} & 0.0 & 11.90 & 11.67 & 5.26 & 6.29 & 2.26 & 1.85 & 3.87 & 3.35 \\
\hline & 2.5 & 15.62 & 12.74 & 6.36 & 7.04 & 2.45 & 1.81 & 4.64 & 4.24 \\
\hline & 5.0 & 15.33 & 12.55 & 5.93 & 6.43 & 2.59 & 1.95 & 4.41 & 4.06 \\
\hline \multirow[t]{3}{*}{2000} & 0.0 & 11.66 & 10.99 & 5.13 & 5.75 & 2.27 & 1.91 & 3.43 & 2.82 \\
\hline & 2.5 & 14.85 & 12.15 & 6.09 & 6.17 & 2.44 & 1.97 & 4.46 & 3.89 \\
\hline & 5.0 & 14.48 & 11.58 & 5.86 & 6.09 & 2.47 & 1.90 & 3.95 & 3.28 \\
\hline \multirow[t]{3}{*}{4000} & 0.0 & 9.80 & 10.76 & 4.08 & 5.38 & 2.40 & 2.00 & 3.11 & 2.31 \\
\hline & 2.5 & 12.85 & 11.91 & 5.92 & 5.87 & 2.17 & 2.03 & 4.23 & 3.62 \\
\hline & 5.0 & 12.66 & 11.48 & 5.83 & 5.70 & 2.17 & 2.01 & 3.87 & 2.73 \\
\hline \multirow[t]{3}{*}{6000} & 0.0 & 9.20 & 10.35 & 4.20 & 4.70 & 2.19 & 2.20 & 3.07 & 2.27 \\
\hline & 2.5 & 12.55 & 11.75 & 5.78 & 5.49 & 2.17 & 2.14 & 4.23 & 3.19 \\
\hline & 5.0 & 12.37 & 10.84 & 5.72 & 5.31 & 2.16 & 2.04 & 3.34 & 2.72 \\
\hline \multirow[t]{3}{*}{8000} & 0.0 & 8.92 & 8.21 & 3.03 & 3.97 & 2.94 & 2.07 & 2.95 & 2.03 \\
\hline & 2.5 & 10.98 & 10.16 & 5.72 & 4.49 & 1.92 & 2.27 & 3.60 & 2.95 \\
\hline & 5.0 & 10.31 & 9.66 & 5.29 & 4.38 & 1.95 & 2.21 & 3.26 & 2.50 \\
\hline \multicolumn{2}{|l|}{ LSD $_{5 \%}$} & \multicolumn{2}{|l|}{0.14} & \multicolumn{2}{|l|}{0.10} & \multicolumn{2}{|l|}{0.05} & \multicolumn{2}{|l|}{0.07} \\
\hline \multicolumn{10}{|c|}{ Mean of main effects } \\
\hline \multirow[t]{2}{*}{ Variety } & DS-9004 & \multicolumn{2}{|l|}{12.23} & \multicolumn{2}{|l|}{5.35} & \multicolumn{2}{|l|}{2.30} & \multicolumn{2}{|l|}{3.76} \\
\hline & LP-15 & \multicolumn{2}{|l|}{11.12} & \multicolumn{2}{|l|}{5.54} & \multicolumn{2}{|l|}{2.02} & \multicolumn{2}{|l|}{3.06} \\
\hline F sig. & & a & & a & & a & & a & \\
\hline Salinity (ppm) & 320.0 & 13.30 & & 6.22 & & 2.15 & & 4.10 & \\
\hline & 2000.0 & 12.62 & & 5.85 & & 2.16 & & 3.64 & \\
\hline & 4000.0 & 11.58 & & 5.46 & & 2.13 & & 3.31 & \\
\hline & 6000.0 & 11.18 & & 5.20 & & 2.15 & & 3.14 & \\
\hline & 8000.0 & 9.71 & & 4.48 & & 2.23 & & 2.88 & \\
\hline LSD $_{5 \%}$ & & 0.10 & & 0.08 & & 0.04 & & 0.05 & \\
\hline Try.(mM) & 0.0 & 8.62 & & 3.98 & & 1.84 & & 2.43 & \\
\hline & 2.5 & 10.46 & & 4.91 & & 1.78 & & 3.25 & \\
\hline & 5.0 & 10.10 & & 4.71 & & 1.79 & & 2.84 & \\
\hline $\mathrm{LSD}_{5 \%}$ & & 0.08 & & 0.06 & & 0.03 & & 0.04 & \\
\hline
\end{tabular}

ns non significant

${ }^{\mathrm{a}}$ Significant

levels. Accordingly, the greatest chlorophyll a/b ratio $(2.94 \mathrm{mg} / \mathrm{g}$ f.w.) was recorded without tryptophan $(0.0 \mathrm{mM})$ under salinity level at $8000 \mathrm{ppm}$ for DS9004 cultivar. While, the lowest chlorophyll a/b ratio $(1.81 \mathrm{mg} / \mathrm{g}$ f.w.) was obtained with tryptophan $(2.5$ $\mathrm{mM}$ ) under salinity level at 320 for LP-15 cultivar. Tryptophan treatment at $2.5 \mathrm{mM}$ was the most pronounced treatment and caused the highest significant increase in carotenoids content $(4.64 \mathrm{mg} / 100 \mathrm{~g} \mathrm{fw})$ under salinity level at $320 \mathrm{ppm}$ for DS-9004 cultivar.
Increasing salinity level (more than $6000 \mathrm{ppm}$ ) decreased significantly photosynthetic pigment contents of all pre-soaking treatments.

Effect of pre-soaking with tryptophan on root parameters of sugar beet under different salinity levels at harvest (180 days after sowing)

Table 4 indicated that pre-soaking with tryptophan $(2.5 \mathrm{mM})$ was the most pronounced treatment and caused the highest significant increase in root 
Table 4 Effect of soaking two sugar beet seed cultivars with tryptophan and irrigated with different salinity levels on root parameters at harvest (180 days after sowing)

\begin{tabular}{|c|c|c|c|c|c|c|c|}
\hline \multicolumn{2}{|l|}{ Treatments } & \multicolumn{6}{|c|}{ Root parameters at harvest } \\
\hline \multirow[t]{2}{*}{ Salinity (ppm) } & \multirow{2}{*}{$\begin{array}{l}\text { Try. } \\
\text { (mM) }\end{array}$} & \multicolumn{2}{|c|}{ Length $(\mathrm{cm})$} & \multicolumn{2}{|c|}{ Diameter $(\mathrm{cm})$} & \multicolumn{2}{|c|}{ Fresh wt. (g) } \\
\hline & & DS-9004 & $L P-15$ & DS-9004 & LP-15 & DS-9004 & LP-15 \\
\hline \multirow[t]{3}{*}{320} & 0.0 & 24.00 & 21.50 & 16.00 & 15.00 & 145.00 & 174.00 \\
\hline & 2.5 & 26.00 & 24.00 & 20.00 & 20.00 & 207.00 & 208.00 \\
\hline & 5.0 & 25.00 & 20.00 & 19.00 & 18.00 & 181.00 & 193.00 \\
\hline \multirow[t]{3}{*}{2000} & 0.0 & 22.00 & 18.50 & 15.00 & 14.00 & 107.00 & 145.00 \\
\hline & 2.5 & 25.00 & 22.00 & 18.00 & 18.00 & 185.00 & 187.00 \\
\hline & 5.0 & 24.00 & 20.00 & 17.00 & 16.00 & 163.00 & 175.00 \\
\hline \multirow[t]{3}{*}{4000} & 0.0 & 20.00 & 18.00 & 15.00 & 12.00 & 126.00 & 124.00 \\
\hline & 2.5 & 26.00 & 20.00 & 19.00 & 17.00 & 183.00 & 172.00 \\
\hline & 5.0 & 23.00 & 19.00 & 16.00 & 15.00 & 156.00 & 159.00 \\
\hline \multirow[t]{3}{*}{6000} & 0.0 & 19.00 & 18.00 & 13.00 & 12.00 & 95.00 & 120.00 \\
\hline & 2.5 & 24.00 & 19.00 & 16.00 & 16.00 & 151.00 & 165.00 \\
\hline & 5.0 & 21.00 & 18.00 & 14.00 & 14.00 & 141.00 & 147.00 \\
\hline \multirow[t]{3}{*}{8000} & 0.0 & 15.00 & 14.00 & 10.00 & 10.00 & 113.00 & 105.00 \\
\hline & 2.5 & 22.00 & 16.00 & 12.00 & 14.00 & 144.00 & 135.00 \\
\hline & 5.0 & 18.50 & 15.00 & 11.00 & 12.00 & 136.00 & 133.00 \\
\hline \multicolumn{2}{|c|}{$\mathrm{LSD}_{5} \%$} & \multicolumn{2}{|l|}{1.77} & \multicolumn{2}{|l|}{0.15} & \multicolumn{2}{|l|}{5.79} \\
\hline \multicolumn{8}{|c|}{ Mean of main effects } \\
\hline \multirow[t]{2}{*}{ Variety } & DS-9004 & \multicolumn{2}{|l|}{22.30} & \multicolumn{2}{|l|}{15.40} & \multicolumn{2}{|l|}{148.87} \\
\hline & LP-15 & \multicolumn{2}{|l|}{18.87} & \multicolumn{2}{|l|}{14.87} & \multicolumn{2}{|l|}{156.13} \\
\hline \multicolumn{2}{|l|}{ F sig. } & \multicolumn{2}{|l|}{ a } & \multicolumn{2}{|l|}{ a } & \multicolumn{2}{|l|}{ a } \\
\hline \multirow[t]{5}{*}{ Salinity (ppm) } & 320.0 & 23.42 & & 18.00 & & 184.67 & \\
\hline & 2000.0 & 21.92 & & 16.33 & & 160.33 & \\
\hline & 4000.0 & 21.00 & & 15.67 & & 153.33 & \\
\hline & 6000.0 & 19.83 & & 14.17 & & 136.50 & \\
\hline & 8000.0 & 16.75 & & 11.50 & & 127.67 & \\
\hline $\mathrm{LSD}_{5 \%}$ & & 1.29 & & 0.11 & & 4.23 & \\
\hline Try.(mM) & 0.0 & 15.83 & & 11.00 & & 104.50 & \\
\hline & 2.5 & 18.67 & & 14.17 & & 144.75 & \\
\hline & 5.0 & 16.96 & & 12.67 & & 132.00 & \\
\hline $\mathrm{LSD}_{5 \%}$ & & 0.97 & & 0.08 & & 3.17 & \\
\hline
\end{tabular}

ns non significant

${ }^{\mathrm{a}}$ Significant

parameters under different salinity levels. Whereas, the highest value of root length $(26.00 \mathrm{~cm})$ were observed with tryptophan $(2.5 \mathrm{mM})$ under two salinity levels (320 and $4000 \mathrm{ppm}$ ) for DS-9004 cultivar. The highest value of root diameter $(20.00 \mathrm{~cm})$ with tryptophan $(2.5 \mathrm{mM})$ under salinity level at $320 \mathrm{ppm}$ for DS-9004 and LP-15 cultivars, while the significant highest root fresh weight $(208.00 \mathrm{~g} /$ plant $)$ was exhibited with tryptophan $(2.5 \mathrm{mM})$ under level $320 \mathrm{ppm}$ of salinity for LP-15 cultivar. Furthermore, sugar beet was significantly affected by the high level of salinity $(8000 \mathrm{ppm})$ that was significantly decreased in root parameters with all pre-soaking treatments.

Effect of pre-soaking with tryptophan on shoot parameters of sugar beet under different salinity levels at harvest ( 180 days after sowing)

Table 5 showed that pre-soaking with tryptophan $(2.5$ $\mathrm{mM}$ ) significantly increased sugar beet shoot parameters of sugar beet at 180 days after sowing as compared with 
Table 5 Effect of soaking two sugar beet seed cultivars with tryptophan and irrigated with different salinity levels on shoot parameters at harvest (180 days after sowing)

\begin{tabular}{|c|c|c|c|c|c|}
\hline \multicolumn{2}{|l|}{ Treatment } & \multicolumn{4}{|c|}{ Shoot parameters at harvest } \\
\hline \multirow[t]{2}{*}{ Salinity (ppm) } & \multirow{2}{*}{$\begin{array}{l}\text { Try. } \\
\text { (mM) }\end{array}$} & \multicolumn{2}{|c|}{ Length $(\mathrm{cm})$} & \multicolumn{2}{|c|}{ Fresh wt. (g) } \\
\hline & & DS-9004 & LP-15 & DS-9004 & LP-15 \\
\hline \multirow[t]{3}{*}{320} & 0.0 & 26.00 & 30.00 & 222.00 & 200.00 \\
\hline & 2.5 & 29.00 & 32.00 & 299.00 & 213.00 \\
\hline & 5.0 & 28.00 & 31.00 & 279.00 & 203.00 \\
\hline \multirow[t]{3}{*}{2000} & 0.0 & 24.00 & 26.00 & 217.00 & 177.00 \\
\hline & 2.5 & 29.00 & 30.00 & 273.00 & 198.00 \\
\hline & 5.0 & 28.00 & 27.00 & 260.00 & 186.00 \\
\hline \multirow[t]{3}{*}{4000} & 0.0 & 24.00 & 23.00 & 194.00 & 152.00 \\
\hline & 2.5 & 28.00 & 30.00 & 268.00 & 179.00 \\
\hline & 5.0 & 27.00 & 24.00 & 256.00 & 169.00 \\
\hline \multirow[t]{3}{*}{6000} & 0.0 & 23.00 & 18.00 & 189.00 & 148.00 \\
\hline & 2.5 & 25.00 & 27.00 & 230.00 & 173.00 \\
\hline & 5.0 & 24.00 & 22.00 & 223.00 & 158.00 \\
\hline \multirow[t]{3}{*}{8000} & 0.0 & 20.00 & 15.00 & 183.00 & 116.00 \\
\hline & 2.5 & 23.00 & 22.00 & 218.00 & 139.00 \\
\hline & 5.0 & 21.00 & 20.00 & 195.00 & 127.00 \\
\hline \multicolumn{2}{|l|}{$\mathrm{LSD}_{5 \%}$} & \multicolumn{2}{|l|}{0.32} & \multicolumn{2}{|l|}{5.95} \\
\hline \multicolumn{6}{|c|}{ Mean of main effects } \\
\hline \multirow[t]{2}{*}{ Variety } & DS-9004 & \multicolumn{2}{|l|}{25.27} & \multicolumn{2}{|l|}{233.73} \\
\hline & LP-15 & \multicolumn{2}{|l|}{25.13} & \multicolumn{2}{|l|}{169.20} \\
\hline \multicolumn{2}{|l|}{ F sig. } & \multicolumn{2}{|l|}{ a } & \multicolumn{2}{|l|}{ a } \\
\hline \multirow[t]{5}{*}{ Salinity (ppm) } & 320.0 & \multicolumn{2}{|l|}{29.33} & \multicolumn{2}{|l|}{236.00} \\
\hline & 2000.0 & \multicolumn{2}{|l|}{27.33} & \multicolumn{2}{|l|}{218.50} \\
\hline & 4000.0 & \multicolumn{2}{|l|}{26.00} & \multicolumn{2}{|l|}{203.00} \\
\hline & 6000.0 & 23.17 & & 186.83 & \\
\hline & 8000.0 & 20.17 & & 163.00 & \\
\hline $\mathrm{LSD}_{5 \%}$ & & 0.23 & & 4.34 & \\
\hline Try. (mM) & 0.0 & 19.08 & & 149.83 & \\
\hline & 2.5 & 22.92 & & 182.50 & \\
\hline & 5.0 & 21.00 & & 171.33 & \\
\hline $\mathrm{LSD}_{5 \%}$ & & 0.17 & & 3.26 & \\
\hline
\end{tabular}

ns non significant

${ }^{\text {as }}$ Significant

other treatment (tryptophan 0.0 and $5.0 \mathrm{mM}$ ) under the different salinity levels. The highest values of shoot length $(32.00 \mathrm{~cm})$, shoot fresh weight $(299.00 \mathrm{~g} /$ plant $)$ were revealed with tryptophan $(2.5 \mathrm{mM})$ under salinity level at $320 \mathrm{ppm}$ for LP-15 and DS-9004 cultivars, respectively. Concerning the effect of different salinity levels, increasing salinity levels (more than $320 \mathrm{ppm}$ ) caused a significant reduction in shoot parameters of all pre-soaking treatments.
Effect of pre-soaking with tryptophan on root quality parameters of sugar beet under different salinity levels at harvest (180 days after sowing)

Root purity parameters

Increasing salinity level decreased significantly the root purity parameters of sugar beet with all pre-soaking treatments as shown in Table 6. It is also clear that presoaking treatment (tryptophan $2.5 \mathrm{mM}$ ) had a significant effect on root purity parameters of sugar beet under salinity level at $320 \mathrm{ppm}$ as compared with (tryptophan 0.0 and $5.0 \mathrm{mM}$ ). Whereas the highest value of sugar \% $(17.71 \%)$ was obtained with tryptophan $(2.5 \mathrm{mM})$ under salinity level at $320 \mathrm{ppm}$ for DS-9004 cultivar. While LP15 cultivar gave the highest value of juice purity (85.30\%) with tryptophan $(2.5 \mathrm{mM})$ under salinity level at $320 \mathrm{ppm}$.

\section{Impurity parameters}

On the contrary, the data concerning impurity parameters indicated that increasing salinity levels increased significantly the impurity parameters of all pre-soaking treatments (Table 6). On the other hand, impurity parameters tended to decrease by pre-soaking treatments, in contrast, to control (tryptophan $0.0 \mathrm{mM}$ ) which gave the maximum concentrations of impurity parameters. The highest concentrations of $\mathrm{K}^{+}$, (4.64) for DS-9004 cultivar, $\mathrm{Na}^{+}$(9.59), and content of $\alpha$ amino-N (5.83) for LP-15 cultivar were obtained without tryptophan (0.0) under salinity level at $8000 \mathrm{ppm}$. While the lowest concentrations of $\mathrm{K}^{+}(1.91), \mathrm{Na}^{+}(1.91)$ and content of $\alpha$ amino- $\mathrm{N}$ (2.17) were obtained with tryptophan $(2.5 \mathrm{mM})$ under salinity level at $320 \mathrm{ppm}$ for LP-15 cultivar.

\section{Discussion}

The above results revealed that the highest root parameters were obtained with tryptophan $(2.5 \mathrm{mM})$ under salinity level at $320 \mathrm{ppm}$, but the lowest significant increments were noticed when sugar beet plants were subjected to a high level of salinity ( $8000 \mathrm{ppm})$. This reduction in plant growth caused by high salinity due to the osmotic effect and adversely affecting physiological and metabolic processes (Hozayn et al., 2019). These results are consistent with that obtained by Hajiboland et al. (2009) which found that sugar beet had a significant growth under moderate salinity levels. The previous studies showed that pre-soaking in tryptophan has increased the growth of many crops like snap bean plants (El-Awadi et al., 2011); chickpea (Abbas et al., 2013); wheat (Mohite, 2013), and quinoa (Bakry et al., 2016). Regarding photosynthetic pigment, results indicated that the pre-soaking treatments had a significant effect on photosynthetic pigment under different salinity levels at 75 days after sowing as compared with control (tryptophan $0.0 \mathrm{mM}$ ). Also, Kandil et al. (2001) revealed that 
Table 6 Effect of soaking two sugar beet seed cultivars with tryptophan and irrigated with different salinity levels on root quality parameters at harvest (180 days after sowing)

\begin{tabular}{|c|c|c|c|c|c|c|c|c|c|c|c|}
\hline \multicolumn{2}{|l|}{ Treatments } & \multicolumn{4}{|c|}{ Root purity } & \multicolumn{6}{|c|}{ Impurity parameters } \\
\hline \multirow[t]{2}{*}{ Salinity (ppm) } & \multirow{2}{*}{$\begin{array}{l}\text { Try. } \\
\text { (mM) }\end{array}$} & \multicolumn{2}{|l|}{ Sugar \% } & \multicolumn{2}{|l|}{ Purity } & \multicolumn{2}{|l|}{ K } & \multicolumn{2}{|l|}{$\mathrm{Na}$} & \multicolumn{2}{|c|}{ Alfa Amino-N } \\
\hline & & DS-9004 & LP-15 & DS-9004 & LP-15 & DS-9004 & LP-15 & DS-9004 & LP-15 & DS-9004 & LP-15 \\
\hline \multirow[t]{3}{*}{320} & 0.0 & 16.15 & 14.41 & 74.69 & 74.00 & 3.27 & 2.52 & 5.82 & 7.02 & 3.42 & 4.02 \\
\hline & 2.5 & 17.71 & 14.96 & 81.92 & 85.30 & 2.04 & 1.91 & 2.16 & 1.91 & 2.25 & 2.17 \\
\hline & 5.0 & 16.77 & 14.48 & 80.95 & 80.99 & 2.20 & 2.59 & 3.31 & 3.23 & 3.89 & 2.94 \\
\hline \multirow[t]{3}{*}{2000} & 0.0 & 16.13 & 13.10 & 74.03 & 72.14 & 3.48 & 2.52 & 6.74 & 7.83 & 3.56 & 4.29 \\
\hline & 2.5 & 17.25 & 14.95 & 80.67 & 80.97 & 2.22 & 2.19 & 2.44 & 1.99 & 2.39 & 3.00 \\
\hline & 5.0 & 16.15 & 13.81 & 79.34 & 80.28 & 2.57 & 2.47 & 3.69 & 3.30 & 3.07 & 3.08 \\
\hline \multirow[t]{3}{*}{4000} & 0.0 & 15.25 & 12.31 & 71.83 & 69.83 & 3.79 & 3.19 & 7.12 & 8.51 & 3.58 & 4.61 \\
\hline & 2.5 & 15.92 & 13.98 & 78.45 & 80.20 & 2.27 & 2.22 & 3.70 & 3.20 & 3.42 & 3.08 \\
\hline & 5.0 & 15.59 & 13.12 & 78.13 & 76.99 & 2.63 & 2.70 & 3.94 & 3.62 & 3.56 & 3.20 \\
\hline \multirow[t]{3}{*}{6000} & 0.0 & 14.75 & 12.25 & 64.81 & 65.29 & 4.21 & 3.21 & 8.19 & 9.26 & 3.91 & 5.77 \\
\hline & 2.5 & 15.25 & 13.12 & 76.46 & 76.21 & 2.45 & 2.65 & 4.07 & 3.38 & 3.64 & 3.83 \\
\hline & 5.0 & 14.96 & 12.08 & 76.42 & 75.69 & 2.81 & 3.02 & 4.68 & 3.65 & 3.93 & 4.05 \\
\hline \multirow[t]{3}{*}{8000} & 0.0 & 13.62 & 11.58 & 64.00 & 64.16 & 4.64 & 3.82 & 8.32 & 9.59 & 4.67 & 5.83 \\
\hline & 2.5 & 15.23 & 11.98 & 74.19 & 76.19 & 2.66 & 4.00 & 5.76 & 3.41 & 3.75 & 4.45 \\
\hline & 5.0 & 14.46 & 11.71 & 67.38 & 71.59 & 3.10 & 4.03 & 6.27 & 3.97 & 4.28 & 5.21 \\
\hline \multicolumn{12}{|c|}{ Mean of main effects } \\
\hline \multirow[t]{2}{*}{ Variety } & DS-9004 & 15.68 & & 74.88 & & 2.96 & & 5.08 & & 3.55 & \\
\hline & LP-15 & 13.19 & & 75.32 & & 2.87 & & 4.92 & & 3.97 & \\
\hline \multirow[t]{5}{*}{ Salinity (ppm) } & 320.0 & 15.75 & & 79.64 & & 2.42 & & 3.91 & & 3.12 & \\
\hline & 2000.0 & 15.23 & & 77.91 & & 2.58 & & 4.33 & & 3.23 & \\
\hline & 4000.0 & 14.36 & & 75.91 & & 2.80 & & 5.02 & & 3.58 & \\
\hline & 6000.0 & 13.74 & & 72.48 & & 3.06 & & 5.54 & & 4.19 & \\
\hline & 8000.0 & 13.10 & & 69.59 & & 3.71 & & 6.22 & & 4.70 & \\
\hline \multirow[t]{3}{*}{ Try. (mM) } & 0.0 & 11.63 & & 57.90 & & 2.89 & & 6.53 & & 3.64 & \\
\hline & 2.5 & 12.53 & & 65.88 & & 2.05 & & 2.67 & & 2.67 & \\
\hline & 5.0 & 11.93 & & 63.98 & & 2.34 & & 3.31 & & 3.10 & \\
\hline
\end{tabular}

photosynthetic pigments content in sugar beet leaves after 90 days from sowing, significantly affected by varieties. Otherwise, with increasing salinity levels more than $6000 \mathrm{ppm}$, there was a decrease in values of all these parameters. These results are in agreement with Khan et al. (2006) and El-Tantawy et al. (2006) who found that chlorophyll a, b, and carotenoids were reduced by 6000, 10,000, and 14,000 ppm. Dadkhah (2011) who found that photosynthesis of leaves was decreased in two cultivars with increasing salinity. Bakry et al. (2016) mentioned that foliar application of tryptophan under water deficit (skipping irrigation) enhanced the growth by stimulating growth regulators level (IAA) and protecting the photosynthetic apparatus. El-Awadi et al. (2017) who found that chlorophyll a, b, and carotenoids were increased under the effect of IAA, tryptophan as seed priming.
That acts on the imbalance of internal hormones, thus inhibiting plant growth (Younis et al., 2003). Results indicate that the pre-soaking treatments were significantly to alleviate the harmful effect of salinity stress on root and shoot parameters at 180 days after sowing as compared with control (tryptophan $0.0 \mathrm{mM}$ ). It was noticed that a significant decrease has existed in root and shoot parameters with increasing salinity levels. These results agreed with those reported by Jamil and Rha (2007) who indicated that sugar beet plants grown under salinity stress exhibited decreased significantly in root and shoot dry weight with the increase in salt concentration. Khayamim et al. (2014) reported that salinity stress decreased root yield. With regard to the effect of amino acids, El-Desouky et al. (2011) and Abd El-Aal (2012) showed that the amino acids treatment significantly increased growth parameters as diameter and stem length, 
number of branches and leaves/plant, fresh and dry weight of stems and leaves, total leaf area/plant, and specific leaf weight. Abdelraouf (2017) found that increasing salt concentration decreased significantly the growth of the root and shoot of sugar beet seedlings with all presoaking treatments. However, the interaction between salinity and pre-soaking treatments had a significant effect on roots growth.

The most important factors which affect the productivity and quality of sugar beetroots are the percentage of sugar, purity (Abdel-Mawly and Zanouny, 2004). This increment of sugars in sugar beet with pre-soaking considered a direct result of the obtained vigorous growth that is accompanied by high photosynthesis efficiency and high yield with high quality (Abd El-Aal, 2012). In both cultivars, sugar \% was decreased with increasing salinity. A reason for this might be due to the smaller size of cells. It was observed that salt stress increases the level of reducing sugars within the cell several plants belonging to different species (Gupta and Huang, 2014). Under severe salinity stress, sugar percentage was reduced as compared to the control $(320 \mathrm{ppm})$. This reduction could be the result of the depressive effect of high salinity on the process of photosynthesis (Khan et al., 1995). Also, Hajiboland et al. (2009) reported that sugar content of sugar beet increased under mild salinity $(\mathrm{EC}=5.5 \mathrm{dS} \mathrm{m}-1)$ when compared with the control and high levels of salinity and this due to that the salt leads to higher accumulation of sugars in roots. Similar results were reported by (Razavizadeh and Rostami, 2013) in canola and Khayamim et al. (2014) in sugar beet.

The maximum concentrations of impurity parameters that inhibited plant growth were observed without presoaking (tryptophan $0.0 \mathrm{mM}$ ) under salinity level at $8000 \mathrm{ppm}$ and minimum concentrations were observed with tryptophan $(2.5 \mathrm{mM})$ under salinity level at 320 ppm. These results are explained previously by Dadkhah (2005) indicated that the white sugar content is affected negatively significant with increasing root impurities as $\mathrm{K}^{+}, \mathrm{Na}^{+}$, and $\alpha$-amino- $\mathrm{N}$. The accumulation of $\mathrm{Na}^{+}$in plant roots due to depressing the uptake of other anions (Darwesh, 2013 \& Wu et al., 2013). Moreover, the purity of sugar beetroots was increased, simultaneously with the reduction of root contents of impurities with presoaking in tryptophan treatment. The opposite was true with increasing salinity. Similar trends were observed by Masri et al. (2015) found that sucrose, total soluble solids, and purity of sugar beet juice decreased with salinity stress. Also, El-Tantawy et al. (2006) concluded that salinity stress increased the $\alpha-$ amino- $\mathrm{N}$ and $\mathrm{Na}^{+}$concentration in the sugar beet. Also, Hussain et al. (2009) and Khafagy et al. (2009) found that $\mathrm{NaCl}$ at $8-15 \mathrm{ds} / \mathrm{m}$ reduced growth parameters and chlorophyll contents in Cassia absus $\mathrm{L}$., whereas $\mathrm{Na}, \mathrm{Cl}$, and $\mathrm{K}$ contents were increased at a high level of $\mathrm{NaCl}$. Meanwhile, presoaking of seeds in glycine betaine and ascorbic acid partially counteracted the harmful effect of $\mathrm{NaCl}$ on sweet pepper and Sadeghi and Shourijeh (2012) on (Sorghum bicolor L). Feizi et al. (2018) indicated that with higher levels of water salinity molasses sugar, leaf weight, and the concentrations of $\mathrm{Na}, \mathrm{K}$, and a- amino- $\mathrm{N}$ in sugar beet significantly increased.

\section{Conclusions}

The pre-soaking of sugar beet seeds in tryptophan $(2.5$ $\mathrm{mM}$ ) is considered one of a simple tool for reducing the negative effect of salinity stress on sugar beet yield. Whereas the studied parameters were recorded the highest values with tryptophan $(2.5 \mathrm{mM})$ under salinity level at $320 \mathrm{ppm}$ for DS-9004 cultivar.

\section{Abbreviations \\ CRD design: Completely randomized design; TSS: Total soluble solids or sugar; Na: Sodium; K: Potassium; LSD: Least significant difference test}

\section{Acknowledgements}

The authors wish to thank to all the staff members of Field Crops Research Department of the National Research Centre, Dokki, Giza, Egypt, for their excellent technical assistance.

\section{Authors' contributions}

All authors shared in this work. HM analyzed and interpreted the data and contribute to review the manuscript before submitting it to the journal. AA performed the experiments. GS was a major contributor in writing the manuscript. All authors read and approved the final manuscript

\section{Funding}

Not applicable.

Availability of data and materials

All data generated or analyzed during this study are included in this published article

Ethics approval and consent to participate

The manuscript does not contain experiments using animals. The manuscript does not contain human studies.

Consent for publication

Not applicable.

Competing interests

The authors declare that they have no competing interests.

\section{Author details}

${ }^{1}$ Field Crop Research Department, Agricultural and Biological Research Division, National Research Centre, 33 El-Behouth St., (Former El-Tahrir St.) Dokki, Giza 12622, Egypt. ${ }^{2}$ Botany Department, Agricultural and Biological Research Division, National Research Centre, 33 El-Behouth St., (Former El-Tahrir St.) Dokki, Giza 12622, Egypt. ${ }^{3}$ Biological Department, Faculty of Science, Tabuk University, Tayma, Saudi Arabia.

Received: 19 October 2019 Accepted: 14 April 2020

Published online: 01 May 2020

\section{References}

A.O.A.C. Association of Official Analytical Chemists. 16th Edn., Official methods of analysis, Washington, DC. 1995.

Abbas SH, Sohail M, Saleem M, Tariq M, Aziz I, Qammar M, Majeed A, Arif M (2013) Effects of L-tryptophan on plant weight and pod weight in chickpea under rain fed conditions. Science, Technology and Development. 32(4):277-280 
Abd El-Aal MMM (2012) Response of Ananas Melon plants to foliar spray with some natural extracts. Res. J. Agric. \& Biol. Sci. 8(2):201-212

Abdel-Mawly SE, Zanouny I (2004) Response of sugar beet (Beta vulgaris L.) to potassium application and irrigation with saline water. Ass. Univ. Bull. Environ. Res. 7(1):123-136

Abdelraouf EA (2017) Effect of presoaking sugar beet (Beta vulgaris L.) seeds with gibberellic, abscisic or ascorbic acids on alleviation of salinity stress. Alex. Sci. Exch. J 38(1):74-81

Bakry BA, Ibrahim MF, Abdallah MMS, El-Bassiouny HMS (2016) Effect of banana peel extract or tryptophan on growth, yield and some biochemical aspects of quinoa plants under water deficit. International Journal PharmTech Research. 9(8):276-287

Cooke DA, Scott RK. The sugar beet crop. Sciencient Practice published by Chapman and Hall, London.1993; pp: 675.

Dadkhah AR. Response of root yield and quality of sugar beet (Beta vulgaris L.) to salt stress. Iran Agricultural Research. 2005;23-24:33-42.

Dadkhah AR. Effect of salinity on growth and leaf photosynthesis of two sugar beet (Beta vulgaris L.) cultivars. Journal of Agricultural Science and Technology. 2011;13.

Darwesh RS (2013) Improving growth of date palm plantlets grown under salt stress with yeast and amino acids applications. Ann Agric Sci 58(2):247-256

Debez A, Chaibi W, Bouzid S (2001) Effet du NaCl et de régulateurs de croissancesur la germination d'Atriplexhalimus L. Cahiers Agric 10(2):135-138

El-Awadi ME, Dawood MG, Abdel-Baky YR, Hassan EA. Physiological effect of melatonin, IAA and their precursor on quality and quantity of chickpea plants grown under sandy soil conditions. Agricultural Engineering International: CIGR Journal, Special issue. 2017;35-44.

El-Awadi ME, El-Bassiony AM, Fawzy ZF, El-Nemr MA (2011) Response of snap bean (Phaseolus vulgaris $\mathrm{L}$ ) plants to nitrogen fertilizer and foliar application with methionine and tryptophan. Nat Sci 9(5):87-94

El-Bassiouny HMS (2005) Physiological responses of wheat to salinity alleviation by nicotinamide and tryptophan. Int J Agric Biology. 7(4):653-659

El-Desouky SA, Ismaeil FH, Wanas AL, Fathy ESL, AbdEl-All MM, Abd MM (2011) Effect of yeast extract, amino acids and citric acid on physio-anatomical aspects and productivity of tomato plants grown in late summer season. Minufiya J Agric Res 36(4):859-884

El-Gamal IS, Abd El Aal MMM, El Desouky SA, Khedr ZM, Abo Shady KA (2016) Effect of some growth substances on growth, chemical compositions and root yield productivity of sugar beet (Beta vulgaris L.) plant. Middle East J Agri Res 5:171-185

El-Tantawy AA, Arafa AMS, El-Banna AE, Darwesh RSS. Effect of salts stress on growth and development on vitro culture, acclimatization stage on Phoenix dactylifera L. and Arecastrum romanzoffianum Becc. Seedlings in greenhouse, Ph.D. Thesis, Faculty of Agriculture, Cairo Univ. 2006;55 pp.

FAOSTAT. The data set "sugar beet, production quantity (tons)" for Egypt contains data from the year 1961 until 2016. http://www.factfish.com/ statistic-country/Egypt/sugar+beet+production . 2016.

Feizi M, Fallahzade J, Noorshargh P (2018) Sugar beet yield response to different levels of saline irrigation water and leaching in an arid region. J Plant Nutr 41(5):654-663

Franzen D. Salt accumulation processes. North Dakota state Univ., Fargo ND. 2007; 58105 .

Gupta B, Huang B (2014) Mechanism of salinity tolerance in plants: physiological, biochemical, and molecular characterization. Int J Genom:1-18

Hajiboland R, Joudmand A, Fotouhi K (2009) Mild salinity improves sugar beet (Beta vulgaris L.) quality. Acta Agric Scand Sec B Soil Plant Sci 59(4):295-305

Hozayn M, Ahmed AA, El-Saady AA, Abd-Elmonem AA (2019) Enhancement in germination, seedling attributes and yields of alfalfa (Medicago sativa, L.) under salinity stress using static magnetic field treatments. Eur Asian J BioSci 13(1):369-378

Hussain K, Majeed A, Nisar MF, Nawaz K, Bhatti KH, Afghan S (2009) Growth and ionic adjustments of chaksu (Cassia absus L.) under $\mathrm{NaCl}$ stress. Am Eur J Agric Environ Sci 6(5):557-560

Hussein MM, Faham SY, Alva AK (2014) Role of foliar application of nicotinic acid and tryptophan on onion plants response to salinity stress. J Agric Sci 6(8): $41-51$

Jamil M, Rha ES (2007) Gibberellic acid (GA3) enhance seed water uptake, germination and early seedling growth in sugar beet under salt stress. Pakistan J Biol Sci 10(4):654-658
Kandil SA, Abo El-Khier MS, Abo-ElLiell AA (2001) Physiological response of some sugar beet (Beta vulgaris L.) genotypes to irrigation with different chloride salinity. Bull NRC Egypt 26(1):76-92

Khafagy MA, Arafa AA, El-Banna MF (2009) Glycinebetaine and ascorbic acid can alleviate the harmful effects of $\mathrm{NaCl}$ salinity in sweet pepper. Aust J Crop Sci 3(5):257

Khan A, Ahmad MSA, Athar HUR, Ashraf M (2006) Interactive effect of foliarly applied ascorbic acid and salt stress on wheat (Triticum aestivum L.) at the seedling stage. Pak J Bot 38:1407-1414

Khan AH, Ashraf MY, Naqvi SSM, Khanzada B, Ali M (1995) Growth and ion and solute contents of sorghum grown under $\mathrm{NaCl}$ and $\mathrm{Na} 2 \mathrm{SO} 4$ salinity stress. Acta Physiol Plant 17:261-268

Khayamim S, Tavakkol Afshari R, Sadeghian SY, Poustini K, Rouzbeh F, Abbasi Z (2014) Seed germination, plant establishment, and yield of sugar beet genotypes under salinity stress. J Agric Sci Technol 16:779-790

Masri MI, Ramadan BSB, El-Shafaiand AMA, El-Kady MS (2015) Effect of water stress and fertilization on yield and quality of sugar beet under drip and sprinkler irrigation systems in sandy soils. Int J Agric Sci 5(3):414-425

Memon YM, Khan I, Panhwar RN (2004) Adoptability performance of some exotic sugar beet varieties under agro-climatic conditions of Thatta. Pakistan Sugar J. 19(6):42-46

Mohamed HF. Chemical and technological studies on sugar beet. Ph.D. Thesis, Faculty of Agriculture, Minia University, Egypt. 2002.

Mohite B (2013) Isolation and characterization of indole acetic acid (IAA) producing bacteria from rhizospheric soil and its effect on plant growth. J Soil Sci Plant Nutr. 13(3):638-649

MSTAT-C program. A software program for the design, management and analysis of Agronomic research experiments. Michigan State University, USA. 1983.

Rai VK (2002) Role of amino acid in plant responses to stresses. Biol Plantarum J. 45:481-487

Razavizadeh R, Rostami F (2013) Changes in growth and antioxidant capacity of canola by salinity and salicylic acid under in vitro

Rezaee Z, Chehrazi M, Moalemi N (2012) Effect of Salinity Stress on Seed Germination Catharanthusroseus Don. Cvs. Rosea and Alba. Asian J Agric Sci. 4(2):117-121

Sadeghi H, Shourijeh FA (2012) Salinity induced effects on growth parameters, chemical and biochemical characteristics of two forage sorghum (Sorghum bicolor L.) cultivars. Asian J Plant Sci 11(1):19-27

Saranya N (2017) Seed priming studies for vigour enhancement in onion CO onion (5). J Pharm Phytochem 6(3):77-82

Wu G, Liang N, Feng R, Zhang J (2013) Evaluation of salinity tolerance in seedlings of sugar beet (Beta vulgaris L.) cultivars using proline, soluble sugars and cation accumulation criteria. Acta Physiologiae Plantarum. 35: 2665-2674

Younis M, El-Shahaby O, Alla MMN, El-Bastawisy Z (2003) Kinetin alleviates the influence of waterlogging and salinity on growth and affects the production of plant growth regulators in Vignasinensis and Zea mays. Agronomie. 23(4): 277-285

\section{Publisher's Note}

Springer Nature remains neutral with regard to jurisdictional claims in published maps and institutional affiliations.

\section{Submit your manuscript to a SpringerOpen ${ }^{\circ}$ journal and benefit from:}

- Convenient online submission

- Rigorous peer review

- Open access: articles freely available online

- High visibility within the field

- Retaining the copyright to your article

Submit your next manuscript at $>$ springeropen.com 